\title{
6 Implicações psicológicas da experiência informal de cuidar
}

\author{
| Margarida Sotto Mayor'; Manuela Leite
}

\section{RESUMO}

O estudo da prestação informal de cuidados a pessoas com diagnóstico de demência e dependentes tem vindo, progressivamente, a ganhar relevo como objecto de estudo para a comunidade científica portuguesa. Com o rápido envelhecimento da população espera-se que o aumento de casos de demência duplique nos próximos 20 anos. Actualmente sem cura conhecida, esta doença, de evolução progressiva, requer uma abordagem reabilitativa que enfatize a manutenção das capacidades existentes e se oriente por diversas grandes áreas. As autoras, com larga experiência de abordagem multidisciplinar desta problemática, tendo seleccionado o período de tempo compreendido entre 2002 e 2011 de publicações sobre "caregiving dementia", apresentam uma aproximação à revisão sistemática analisando o conteúdo de vários artigos. Os resultados globais indicam uma actuação centrada na reabilitação da díade cuidador-idoso promovendo melhorias nos aspectos comunicacionais desta díade, reduzindo deste modo as implicações psicológicas associadas à actividade informal de cuidar.

\section{PALAVRA-CHAVE: Cuidadores, demência, implicações psicológicas, ansiedade, depressão, coping}

\begin{abstract}
The study of informal care for people with dementia and their dependents has been gradually gaining importance as an object of study for the Portuguese scientific community. With the rapidly aging population is expected that the increase in cases of dementia will double within the next 20 years. Currently with no known cure, this progressive disease requires a rehabilitative approach that emphasizes the maintenance of existing capabilities and that can be guided through several major areas. The authors, with extensive experience of a multidisciplinary approach to this problem, having selected the time period between 2002 and 2011 of publications on "dementia caregiving," present a systematic approach to analyzing the content review of several articles.The overall
\end{abstract}

results indicate a performance focused on the rehabilitation of elderly-caregiver dyad promoting improvements in the communication of the dyad, thus reducing the psychological implications associated with the activity of informal care.

\section{KEYWORDS: Caregivers, dementia, psychological implications, anxiety, depression, coping}

\section{INTRODUÇÃO}

Os cuidadores, familiares ou não, são figuras centrais para a pessoa que padece de demência e a sua importância relaciona-se com a globalidade da pessoa. O papel por si desempenhado ultrapassa de sobremaneira a satisfação das necessidades básicas e é encarado pelas equipas de saúde como pedra basilar no esclarecimento do diagnóstico, no acompanhamento, na avaliação/intervenção e consecução dos planos terapêuticos.

O conhecimento pela equipa de saúde dos mecanismos de coping (Au et al., 2010), habitualmente usados pelo cuidador, potencia a intervenção partilhada e permite identificar situações que reclamam intervenção atempada. Sabese que estratégias de coping centradas no problema têm mais sucesso que as estratégias centradas nas emoções (Tschanz et al., 2010). São múltiplas as formas de demência o que se associa com maiores dificuldades de diagnóstico e maiores dificuldades para assumir perante a pessoa ou o seu familiar o aparecimento desta patologia com os riscos que este tipo de informação pode despoletar (Draper, Peisah, Snowdon, \& Brodaty, 2009). Consequentemente há também mais necessidade de esclarecimento ao cuidador (Rocca et al., 2011). A experiência de cuidar requer tarefas adaptativas pelas implicações psicológicas que provoca. Habitualmente o diagnóstico é feito numa fase já avançada da doença mas segundo Jansen et al. (2011), nem sempre é benéfico para o cuidador conhecer precocemente o percurso da doença.

A reversibilidade de algumas demências leva a que as equipas de saúde intervenham rapidamente com o cuidador para repor a normalidade da pessoa e reduzir ao máximo os défices resultantes da presença de elementos responsáveis

${ }^{1}$ Enfermeira, Especialista em Enfermagem de Saúde Mental e Psiquiatrica, Serviço de Psicogeriatria do Hospital Magalhães Lemos /Unidade de Investigação e Formação sobre Adultos e Idosos

2 Psicóloga, Doutora, Departamento de Psicologia, Instituto Superior de Ciências da Saúde do Norte, mgsottomayor@gmail.com Submetido em 29-03-2011. Aceite em 14-05-2011.

Citação: Sotto Mayor, M. \& Leite, M. (2011). Implicações psicológicas da experiência informal de cuidar. Revista Portuguesa de Enfermagem de Saúde Mental. 5, 37-44. 
pelas alterações. O conhecimento da irreversibilidade de outras demências, como acontece, por exemplo, com as de origem vascular e que surgem na sequência de AVCs, conduz a equipa de saúde às atitudes de formação que visam a prevenção das potenciais demências integrando no acompanhamento de consultoria a importância da vigilância de saúde em geral e, particularmente, nas pessoas idosas. Perante processos demenciais de características não preveníveis, a atitude que deve nortear as equipas de saúde é relevante para permitir melhor qualidade de vida aos que deles possam padecer e àqueles que voluntariamente ou por falta de opção assumiram o nobre papel de cuidar (Ducharme, Lévesque, Lachance, Kergoat, \& Coulombe, 2011). Sabe-se que no envelhecimento há diferenças individuais marcadas, nos padrões de declínio cognitivo e de identidade (Caddell \& Clare, 2009), relacionados com aspectos socioeconómicos, de escolaridade, de saúde física e outros, sendo necessário cuidado na análise dos resultados individuais. A relevância da história clínica, do exame clínico, da avaliação neuropsicológica e funcional indica o cuidador principal como parceiro no diagnóstico e elemento fulcral para viabilizar o plano de intervenção. O cuidador faz parte integrante do sucesso das intervenções farmacológicas ou não farmacológicas (Sotto Mayor, 2009). A escuta empática do cuidador permite identificar as suas dificuldades, reduzir riscos (Douglas, Letts, \& Richardson, 2011), reconhecer momentos de satisfação, perceber o coping utilizado pelas famílias e potenciais alterações psicológicas, sendo um meio privilegiado para prevenir o aparecimento de sintomatologia depressiva. Todas as intervenções com a pessoa que padece de demência exigem a atenção do cuidador. Diversos estudos demonstram que existe correlação positiva entre os sintomas comportamentais e psicológicos na demência e a redução da funcionalidade com sensação de sobrecarga por parte do cuidador (Miyamoto, Tachimori, \& Ito, 2010) e o sentimento de sobrecarga. A equipa de saúde, na posse desta informação, para todos, inquestionável, deve tomar consciência que da sua intervenção com os cuidadores resultará o sucesso ou não, para todos os envolvidos. Com este trabalho, as autoras pretendem produzir um texto em torno das implicações psicológicas associadas à actividade de cuidar através de uma aproximação à revisão sistemática sobre "caregiving".

\section{MÉTODOS E TÉCNICAS}

Foi efectuada uma pesquisa na Elsevier e seleccionados da base de dados 9609 abstracts, referentes ao período de publicação compreendido entre 2002 e 2011, tendo como condição de procura, em todas as fontes e em todas as ciências, a temática de "caregiving". Seguiu-se uma procura mais específica considerando-se apenas os abstracts alusivos a "caregiving dementia" num total de 1624 ficando apenas com textos relacionados com cuidadores de doentes que padeciam de demência. Com base numa análise qualitativa destes 1624 abstracts foram seleccionados apenas
64 textos integrais, originais, tendo como critérios de selecção uma abordagem multidisciplinar da actividade de cuidar e a assumpção do acompanhamento dos cuidadores como condição inerente ao bem-estar da díade cuidador-idoso. Depois desta primeira análise de conteúdo foram escolhidos todos os textos que referiam "as implicações psicológicas do acto de cuidar". Com referência a estas leituras e posteriores análises foi efectuada a produção deste texto de revisão subdividindo-o em "implicações psicológicas da experiencia informal de cuidar", "implicações psicológicas", "coping e cuidar" e "estratégias de intervenção".

\section{RESULTADOS/DISCUSSÃO}

Subjacente à análise de conteúdo efectuada é assumida a posição pessoal das autoras da investigação face à temática em estudo bem como dos seus percursos profissionais de cuidadores formais e como investigadoras (Serrano, 1996). Este trabalho, tendo a realidade como premissa orientadora, não abarca toda a literatura publicada sobre o tema mas, obedece aos critérios predefinidos pelas investigadoras. Teve em conta a diversidade metodológica usada nos diferentes estudos, a solidez dos respectivos desenhos de investigação e o tipo e características das populações em análise.

Os diversos estudos sobre cuidadores informais indicam que estes estão sujeitos a grande sobrecarga (Garcés, Carretero, Ródenas, \& Sanjosé, 2009). Para alguns autores trata-se mesmo de uma situação que ultrapassa a sua capacidade física e mental com vivência de grandes períodos de stress (Zarit, Charles, \& Irene, 2008). É uma actividade que tem custos directos e indirectos com graves implicações sociais, problemas de saúde já devidamente identificados, como depressão, ansiedade, distúrbios psicossomáticos, alterações imunológicas e alterações cardiovasculares (Yee \& Schulz, 2000; Lee et al., 2003).

Alguns autores mencionaram também o desconforto emocional vivido pelos cuidadores e o impacto que esta vivência tem na pessoa alvo dos cuidados sendo que muitas vezes sofre uma queda na assistência, estando também mais sujeita a negligência e consequentemente à mercê de uma institucionalização precoce.

Por outro lado, a sobrecarga económica a que estas famílias estão sujeitas aumenta também a sensação de impotência pessoal levando estes cuidadores a ser consumidores do sistema de saúde, pondo em causa a sustentabilidade dos sistemas de protecção social e de saúde das gerações vindouras (Garcés, Carretero, Ródenas, \& Alemán, 2010). 


\section{IMPLICACCÕES PSICOLÓGICAS DA EXPERIÊNCIA INFORMAL DE CUIDAR}

São múltiplas as implicações psicológicas que resultam da experiência de cuidar. $O$ confronto com o diagnóstico de demência num familiar, requer da parte da pessoa cuidadora algumas tarefas adaptativas entre elas as relacionadas especificamente com a doença tais como: lidar com a dor, incapacidade e outros sintomas do doente; lidar com o ambiente hospitalar e procedimentos especiais de tratamento que têm de aprender; desenvolvimento e manutenção de um relacionamento adequado com a equipa de saúde -e tarefas mais gerais onde se incluem: a preservação de um equilíbrio emocional razoável (Chang, Chiou, \& Chen, 2010), perante as perdas a enfrentar nomeadamente do familiar; preservação de uma auto-imagem satisfatória e manutenção de um sentido de competência e de mestria; manutenção dos relacionamentos com familiares e amigos tendo em consideração a tendência para $\mathrm{o}$ isolamento e a preparação para um futuro incerto, não esquecendo o carácter degenerativo e progressivo das demências embora com apresentações diversificadas (Rocca et al., 2011). A componente de informação é uma preciosa ajuda para auxiliar o cuidador a lidar com a doença e com a progressiva incapacidade física e cognitiva associadas. Para tal, o fornecimento de informação (acerca das "situaçõesproblema" resultantes das reacções dos doentes com esta patologia, já tipificadas, organizadas e conhecidas dos técnicos), pode ser um elemento chave para gerir com eficácia e eficiência a procura de assistência médica.

A prestação de cuidados a um familiar, tem um impacto negativo no bem-estar físico e emocional do cuidador (Monin \& Schulz, 2009), repercutindo-se em múltiplas esferas da sua vida, quer no âmbito social e económico, quer no âmbito da saúde física e mental. Neste contexto, e de acordo com Garcia (2010), estas podem ser directas (e.g. dores nas costas) ou indirectas (e.g. perda de oportunidades profissionais); explícitas (e.g. conflitos familiares), ou implícitas (e.g. motivação), considerando-se que os efeitos sobre a saúde mental são os mais significativos, comparativamente à saúde física do cuidador.

Do ponto de vista da saúde mental são vários os autores que referem, o stress (Boerner, Schulz, \& Horowitz, 2004; Garcia, 2010; Musil, Morris, Warner, \& Saeid, 2003) a ansiedade (Sala, 2002), e a depressão (Garcia, 2010; Loss, Change \& Grief, 2003) como as consequências directas mais prevalentes nos cuidadores de familiares com demência.

Alguns autores (Baltar, Cerrato, Fernández de Trocóniz e González, 2006) referem ainda os sentimentos de culpa, a raiva, o desamparo, a frustração, a ira, a solidão e a negação de necessidade de ajuda, como os aspectos emocionais e comportamentais relevantes, presentes nos cuidadores. A ira e a hostilidade são ainda reforçadas por Marks, Lambert e Choi (2002).

Garcia (2010) também nos alerta para o facto de estar subjacente à prestação de cuidados um conjunto de consequências (físicas, emocionais, económicas e sociais) que interagem entre si, criando sinergias que podem ser negativas (e.g. cansaço físico, stress e depressão, diminuição dos rendimentos e das interacções sociais) ou positivas (e.g. aumento da actividade física, reconhecimento social e familiar). Do ponto de vista psicológico, o desenvolvimento da empatia (compreensão do sofrimento dos outros), satisfação e sensação de controlo, representam os aspectos positivos essenciais, assim como a "aprendizagem vital" (Crespo \& López, 2004, citado por Garcia, 2010).

A sobrecarga, ou o stress, oriundos da prestação de cuidados não são percepcionados igualmente por todos os cuidadores, estando dependentes das características da personalidade, dos comportamentos do doente, do grau de parentesco com o doente, do sexo do cuidador, do suporte social, e das estratégias de coping utilizadas. Losada-Baltar \& Montorio-Cerrato (2005), referem ainda as crenças e pensamentos disfuncionais e Crespo, López, \& Zarit (2005) a auto-estima e o sentimento de auto-eficácia. A emissão de comportamentos disruptivos por parte dos doentes (Mittelman, Roth, Haley, \& Zarit, 2004), a existência de um laço de familiaridade (Sala, 2002), e a exposição constante ao sofrimento físico e psicológico da pessoa amada, podem influenciar directamente a experiência emocional dos cuidadores (Monin \& Schulz, 2009). A comprovar a importância do apoio social como variável preditiva da saúde mental, surgem-nos os resultados dos estudos desenvolvidos por Leite, Guerra e Garrett (2007).

Crenças e pensamentos disfuncionais (e.g. "seria egoísta cuidar de mim mesma já que é o meu familiar que está doente"; "não devo pedir ajuda aos meus familiares, já que estes têm a sua vida própria e problemas"), podem condicionar o desenvolvimento adequado de estratégias de coping, sendo necessárias intervenções no sentido de promover o desenvolvimento de comportamentos que facilitem a adaptação à prestação de cuidados, como por exemplo, pedir ajuda (Losada-Baltar \& Montorio-Cerrato, 2005).

Crespo López e López Martínez (2007) explicam que «recursos e estratégias son elementos de los que el cuidador dispone para tratar de amortigar el posible impacto de la situación de cuidado" (p. 48). No entanto, apesar de Lazaun, Arrazola, \& Arrazola (2001) considerarem o apoio social e as estratégias de coping, como variáveis modeladoras e modificadoras do stress da prestação de cuidados, advertemnos para a inexistência de um padrão único de adaptação e coping.

\section{COPING E CUIDAR}

O apoio social e as estratégias de coping surgem na literatura como as variáveis moduladoras e modificadoras do stress oriundo da prestação contínua de cuidados (Lazaun, Arrazola, \& Arrazola, 2001). 
O coping define o conjunto de sentimentos, pensamentos e comportamentos que permitem ao indivíduo manter um estado psicológico satisfatório quando ele é ameaçado (Ramos, 2005).

Trata-se de um processo que é simultaneamente emocional e cognitivo e a partir do qual o indivíduo avalia acerca dos potenciais efeitos dos acontecimentos no seu bem-estar ( $R$. S. Lazarus \& Folkman, 1984). Envolve tarefas adaptativas e, perante as exigências internas e externas, as pessoas são postas à prova. De acordo com os autores, ao coping são geralmente atribuídas duas funções primordiais: regular emoções desajustadas e alterar a situação ou o problema, classificando as estratégias de coping, como centradas nas emoções ou centradas no problema.

É assim que a pessoa centrada na emoção, e com referência aos esforços para diminuir a tensão, pode auto punir-se, culpabilizar-se, distanciar-se ou evidenciar fuga ao problema que a aflige, procurar ajuda na sua rede de suporte, aceitar a responsabilidade e papéis, fazer uma reavaliação positiva da situação ou problema, desvalorizar a situação que parece ameaçadora.

Outra hipótese, será centrar-se na resolução do problema e agir sobre a natureza da perturbação, ter percepção alternativa sobre a situação-problema e, no sentido de a alterar, ser por vezes mais pró-activo e agir ou usar de mais racionalidade tendo como objectivo central a sua resolução.

Quando as situações-problema são susceptíveis de mudança, o coping, mais frequentemente adoptado, centrase na resolução de problemas. Quando estão em causa situações avaliadas como sendo de difícil solução, como a inevitabilidade de uma cirurgia, por exemplo, ou ter de cuidar até à morte de uma pessoa que padece de demência, é mais usado o coping centrado nas emoções.

Relacionando os vários tipos de estratégias de coping com o sexo do cuidador, verifica-se a utilização predominante de estratégias confrontativas por parte das mulheres, comparativamente com os homens, que preferem estratégias de fuga ou evitamento (Mackey, Diemer, \& O×Brien, 2000).

López (2008) defende que o facto de os homens cuidadores encararem a prestação de cuidados de uma forma "profissional", permite-lhes ter um maior domínio e controlo das situações. Em situações de demência, as estratégias de confronto, podem revelar-se contraproducentes, potenciar no doente um aumento significativo do stress vivencial, podendo inclusive culminar com alterações de comportamento. Estratégias de "validação" (mostrar-se cooperante com a atitude/pensamento do doente, dominando e conduzindo a situação a seu favor) são mais bem sucedidas.

Parece central a ideia do dinamismo subjacente à tomada de decisões, resultante da avaliação que a pessoa faz da situação, dos obstáculos à resolução do mesmo, e dos seus recursos (internos ou externos). Neste sentido, a inexistência de um padrão único de coping, resulta da consciência de que, um acontecimento ou situação poderá ser stressante para um determinado indivíduo mas não o ser para outro, dependendo dos seus recursos internos, externos, valores culturais e experiências vivenciais. Neste contexto, tanto o processo como as estratégias de coping assumem uma natureza eminentemente transaccional, isto é, pessoa e ambiente estão em permanente interacção, a qual se apresenta como dinâmica, mútua, recíproca e bidireccional (Fonseca, 2005). Ainda segundo este autor o aspecto contextual interfere na adopção de determinadas estratégias em detrimento de outras.

Perante os problemas ou situações, a apreciação ou a avaliação cognitiva da situação e as reacções do indivíduo para lhe fazer face constituem processos mediadores entre a pessoa e o seu meio ambiente (Fonseca, 2005). Esta apreciação cognitiva é um processo de julgamento em que a pessoa avalia se um possível agente stressante é relevante, ou não, para o seu bem-estar e em caso afirmativo, se é potencialmente bom ou mau (Ribeiro, 1998).

A apreciação de um acontecimento como benigno-positivo, por parte do cuidador, ocorre se as consequências são valorizadas como positivas, o mesmo será dizer, se preservam ou promovem o bem-estar, ou se parecem ajudar a consegui-lo. Estas apreciações caracterizam-se por gerar emoções agradavéis, tais como, alegria, amor, felicidade ou tranquilidade (R. Lazarus, 1991, 2000; R. S. Lazarus \& Folkman, 1984). Com esta avaliação a pessoa assegura que a auto-estima ou o auto conceito, valores e objectivos, não serão postos em causa. Os acontecimentos ameaçadores referem-se a danos ou perdas que, embora não tenham ocorrido, são previsíveis. Mesmo que já tenham ocorrido, podem ser igualmente considerados como ameaça pela carga de implicações negativas que essa perda traz consigo (R. Lazarus, 1991). O dano significa que as exigências do meio ultrapassam a capacidade da pessoa para lidar com elas: numa situação de dano, é como se já tivéssemos perdido (Ramos, 2001, 2005).

Nestas apreciações reflectem-se traços de personalidade, locus de controlo, expectativas de auto-eficácia, afectividade, auto-estima, estilos de coping, suporte social, crenças e valores pessoais. Também tem influência, para a avaliação das situações tidas como stressantes, o neuroticismo da pessoa e a tendência para experimentar emoções negativas como resposta às situações (Hooker, Monahan, Shifren, \& Hutchinson, 1992). Predomina o foco da atenção nas reacções internas e o recurso a estratégias de coping menos eficazes, sendo o evitamento (Olshevski, Katz, \& Kight, 1999), uma delas e com consequências para o receptor de cuidados.

Reportando-nos à prestação de cuidados, a situação de doença e dependência funcional de um familiar pode ser avaliada como uma ameaça, um desafio ou uma perda. 
Dependendo das características de cada pessoa, do seu projecto de vida, da fase em que se encontra e da cultura em que está inserida (Helman, 2003), a situação de cuidado (e.g. uma situação que ameaça o seu bem-estar) poderá assim, apresentar-se como desafiante, ou ainda pode significar uma perda para a pessoa. Como dissemos atrás, a avaliação que se faz está muito dependente dos recursos internos e externos dos cuidadores, que se vão alterando ao longo da vida, o que indica que cada caso terá os seus resultados e desfechos próprios.

Alguns autores (Moos, 1984; Paúl \& Fonseca, 2005), que vêem no cuidado uma actividade que gera sobrecarga, referem que a situação de doença prolongada de um familiar pode ser interpretada como uma situação de crise geradora de stress.

Alguns cuidadores, de fracos recursos pessoais e sociais, passam por processos de doença e desequilíbrio emocional, na sequência de tempo prolongado de cuidado, culpabilizando-se pela situação que vivem e por não poderem, simultaneamente, alterar ou modificar as dificuldades do seu dia-a-dia. Perante situações adversas, na ausência de mecanismos de resolução de problemas imediatos, ficam sujeitos a situações de alguma gravidade, que podem conduzir a um estado de desorganização psico-emocional e social, frequentemente acompanhado de sentimentos negativos, como o medo, a ansiedade e a culpa.

A utilização de estratégias antecipatórias no cuidado às pessoas com demência tem-se mostrado bastante eficaz para prevenir, situações de agitação ou de confusão nos doentes nas diversas fases da sua patologia. Um exemplo que reflecte a importância deste tipo de estratégias é retirar a chave da porta se a pessoa a cuidar tem tendência para fuga. Esta atitude previne momentos de sofrimento e de culpa para os cuidadores.

O coping orientado para as emoções (R. S. Lazarus \& Folkman, 1984), no caso dos cuidadores, também pode ser uma forma de viver a situação que dificilmente se alterará. Cuidar, acreditando que a pessoa que está a cargo não tem nem culpa da situação, nem dos comportamentos que assume, e relativizar a experiência vivida (Nolan, Grant, \& Keady, 1998), ajuda os cuidadores a continuar a tarefa com esperança, uma vez que em termos cognitivos pode não haver mais nada a fazer (Nolan et al., 1998). Tudo isto exige um reformular da situação que culmina com a regulação da resposta emocional do indivíduo às exigências (R. S. Lazarus \& Folkman, 1984).

\section{ESTRATÉGIAS DE INTERVENÇÃO}

O seguimento sistemático do doente/família por uma equipa de saúde facilita orelacionamento adequado entrecuidadores, doentes e técnicos tornando menos problemática a procura de cuidados de saúde. Em relação às tarefas adaptativas mais gerais, como a preservação do equilíbrio (perante as perdas que na situação de demência são consecutivas), o sentido de competência e de mestria (apesar dos repetidos insucessos), e a manutenção dos vínculos sócio-afectivos (não obstante o elevado número de horas a cuidar), estão fortemente ligadas com as características biológicas, sociais e culturais, e com os recursos internos e externos de cada pessoa e o modo como habitualmente reage em situações de desprazer. De qualquer modo, a experiência informal de cuidar contempla implicações psicológicas que resultam da "perda da pessoa", com origem no declínio cognitivo e funcional com danos irreparáveis nos aspectos relacionais e, a "perda simbólica", que deriva da impossibilidade de projectar o futuro, de realizar sonhos, em suma, da vivência em família (Mulligan, 2010). O não reconhecimento do cuidador por parte do doente, primeiramente de modo esporádico, depois de modo definitivo contribui decisivamente para esta "perda simbólica" sob a forma de forte impacto psicológico.

Em algumas situações pode até significar a "perda do eu" como resultado da aquisição ou alteração constante dos papéis desempenhados com risco de crise de identidade. Há, como referiu Morton (2004), uma jornada psicológica a percorrer. A fase da negação em que o cuidador assume uma atitude recriminatória face aos défices do doente; a fase do diagnóstico com uma avaliação médica especializada, e a vivência de períodos de angústia associada a uma consciência da irreversibilidade da situação e a entrada na nova situação, a fase da aceitação (procura incessante de informação e a delineação de mudanças na tentativa de gestão dos novos papéis). Na sequência, surge a fase de tentativa de controlo da situação sendo esta caracteristicamente uma fase muito activa e de reestruturação (Lovell \& Wetherell, 2011) inclusive das relações familiares. São necessários muitos ajustes pessoais e familiares e podem surgir conflitos. Com o passar do tempo progride para uma espécie de rendição, sendo um processo habitualmente de grande stress, com avaliação da própria vida, sentimentos de raiva e de desespero, algumas vezes deslocados para familiares ou amigos. Dependendo do grau de parentesco (filhos cônjuges ou outros), surge a necessidade de reaprender a individualidade em contraposição ao "nós" com transformação na pessoa. A jornada psicológica termina com o sentimento de que se está encurralado, é uma altura de tarefas intensas, de cuidados e o momento em que o cuidador precisa de grande ajuda pois está exausto física e psicologicamente. Necessita de reforços positivos para o trabalho até aí desenvolvido. Precisa de se sentir em paz com o papel desempenhado. Esta fase, normalmente evolui para outra que culmina com a separação que pode ocorrer mesmo antes da morte do doente (transferência do doente para instituições especializadas, ou contratação de serviços em casa). Na vivência de Morton (2004), está expresso o turbilhão de sentimentos que pode estar presente em muitos dos cuidadores que atendemos e estudamos. São visíveis os esforços, progressos e retrocessos que qualquer cuidador pode experimentar na procura do equilíbrio psicológico que Ihe permita continuar a cuidar. 


\section{CONCLUSÕES}

Dado o carácter vasto e geral do conteúdo de uma revisão, encerra-se com uma recapitulação dos achados mais pertinentes e com algumas considerações e propostas de intervenção.

Actualmente defendem-se as oportunidades de descanso, grupos de auto ajuda, programas psicoeducativos e programas psicoterapêuticos (individualizados), como as estratégias de intervenção mais eficazes na optimização dos recursos (internos e externos) dos cuidadores, diminuindo o impacto psicológico e o risco de morbilidade associada. No entanto, a sua eficácia implica a participação activa dos cuidadores, sobretudo nos modelos de intervenção baseados no stress e no desenvolvimento de estratégias de coping.

Para além disso, e na linha de pensamento de Carretero \& Garcés (2011), também nós reconhecemos a urgência na revisão das políticas globais que suportam o bem-estar físico e psicológico dos cuidadores informais levando os governos europeus a tomar medidas que visem o sucesso deste grupo de pessoas, com vista a reduzir a sua fragilidade, a sobrecarga a que estão sujeitos sem preparação prévia e sem possibilidade de escolha consciente, de modo a reduzir os encargos com o sistema de saúde e a optimizar os recursos técnicos disponíveis. Da maioria dos textos consultados e investigações realizadas é quase unânime a ideia de que proporcionar temporariamente alívios associados com períodos de formação pessoal tem efeitos benéficos na díade-cuidador idoso. Neste contexto, revela-se crucial uma revisão cuidadosa e especializada dos principais programas psicopedagógicos e sociais utilizados com estes cuidadores com a finalidade de melhorar de forma substancial as práticas de apoio, reduzir as implicações psicológicas e prevenir situações graves de negligência e agravamento recorrente da saúde e bem-estar dos cuidadores informais de pessoas que padecem de demência.

\section{REFERÊNCIAS BIBLIOGRÁFICAS}

Au, A., Li, S., Lee, K., Leung, P., Pan, P.-C., Thompson, L., et al. (2010). The Coping with Caregiving Group Program for Chinese caregivers of patients with Alzheimer's disease in Hong Kong. Patient Education and Counseling, 78(2), 256260.

Baltar, A. L., Cerrato, I. M., Férnandez de Trocóniz, M. I., \& González, M. M. (2006). Estudo y intervención sobre el malestar psicologico de los cuidadores de personas con demencia. El papel de los pensamientos disfuncionales. IMSERSO: Madrid.

Boerner, K., Schulz, R., \& Horowitz, A. (2004). Positive Aspects of Caregiving and Adaptation to Bereavement. Psychology and Aging, 19(4), 668-675.
Caddell, L. S., \& Clare, L. (2009). The impact of dementia on self and identity: A systematic review. Clinical Psychology Review, 30(1), 113-126.

Carretero, S., Garcés, J., Ródenas, F., \& Sanjosé, V. (2008). The informal caregiver's burden of dependent people: Theory and empirical review. Archives of Gerontology and Geriatrics, 49(1), 74-79.

Carretero, S., Garcés, J., Ródenas, F., \& Sanjosé, V. (2009). The informal caregiver's burden of dependent people: Theory and empirical review. Archives of Gerontology and Geriatrics, 49(1), 74-79.

Carretero, S., \& Garcés, J. (2011). P03-04 - Psychological interventions to fight against the burden of the informal caregivers. European Psychiatry, 26(Supplement 1), 1173-1173.

Chang, H.-Y., Chiou, C.-J., \& Chen, N.-S. (2010). Impact of mental health and caregiver burden on family caregivers' physical health. Archives of Gerontology and Geriatrics, 50(3), 267-271.

Crespo, M., \& López, J. (2004). Los costes de cuidar: análisis del estado emocional de cuidadores informales de mayores dependientes. JANO, 1511, 78-79.

Crespo, M., López, J., \& Zarit, S. (2005). Depression and anxiety in primary caregivers: a comparative study of caregivers of demented and non demented older persons. International Journal of Geriatric Psychiatry, 20, 591-592.

Douglas, A., Letts, L., \& Richardson, J. (2011). A systematic review of accidental injury from fire, wandering and medication self-administration errors for older adults with and without dementia. Archives of Gerontology and Geriatrics, 52(1), e1-e10.

Draper, B., Peisah, C., Snowdon, J., \& Brodaty, H. (2009). Early dementia diagnosis and the risk of suicide and euthanasia. Alzheimer's and Dementia, 6(1), 75-82.

Ducharme, F., Lévesque, L., Lachance, L., Kergoat, M.-J., \& Coulombe, R. (2011). Challenges associated with transition to caregiver role following diagnostic disclosure of Alzheimer disease: A descriptive study. International Journal of Nursing Studies.

Fonseca, A. M. (2005). Desenvolvimento humano e envelhecimento. Lisboa: Climepsi Editores.

Garcia, J. (2010). Los tiempos del cuidador: el impacto de la dependencia de los mayores en la vida cotidiana de sus cuidadores. IMSERSO: Madrid.

Garcés, J., Carretero, S., Ródenas, F., \& Sanjosé, V. (2008). Variables related to the informal caregivers' burden of dependent senior citizens in Spain. Archives of Gerontology and Geriatrics, 48(3), 372-379. 
Garcés, J., Carretero, S., Ródenas, F., \& Sanjosé, V. (2009). Variables related to the informal caregivers' burden of dependent senior citizens in Spain. Archives of Gerontology and Geriatrics, 48(3), 372-379.

Garcés, J., Carretero, S., Ródenas, F., \& Alemán, C. (2010). A review of programs to alleviate the burden of informal caregivers of dependent persons. Archives of Gerontology and Geriatrics, 50(3), 254-259.

Garcés, J., Carretero, S., Ródenas, F., \& Vivancos, M. (2010). The care of the informal caregiver's burden by the Spanish public system of social welfare: A review. Archives of Gerontology and Geriatrics, 50(3), 250-253.

Helman, C. G. (2003). Aspectos culturais do estress. Cultura Saúde e doença.

Hooker, K., Monahan, D., Shifren, K., \& Hutchinson, C. (1992). Mental and physical health of spouse caregivers: the role of personality. Psychology and Aging, 7(3), 367-375.

Jansen, A. P. D., van Hout, H. P. J., Nijpels, G., Rijmen, F., Dröes, R.-M., Pot, A.-M., et al. (2011). Effectiveness of case management among older adults with early symptoms of dementia and their primary informal caregivers: A randomized clinical trial. International Journal of Nursing Studies.

Lazarus, R. (1991). Emotion and adaptation. New York: Oxford University Press.

Lazarus, R. (2000). Evolution of a model of stress, coping, and discrete emotions. In V. Rice (Ed) Handbook of stress, coping, and health:implications for nursing, research, theory and practice. London: Sage Publications.

Lazarus, R. S., \& Folkman, S. (1984). Stress, appraisal and coping. New York: Springer Publishing Company.

Lazaun, J., Arrazola, F., Arrazola, M. (2001). Apoio formal y cuidado de las personas dependientes. Retrieved in March, 25, 2011, from http://www.matiaf.net/profissionales/ articullos.

Lee, S.L., Colditz, G.A., Berkman, L.F. \& Kawachi, (2003) Caregiving and risk of coronary heart disease in U.S. women: a prospective study, Am. J. Prev. Med. 24

Leite, M, Guerra, M. \& Garrett, C. (2007). Stress e Prestação de Cuidados a Familiares com Demência de Alzheimer. II Congresso Família, Saúde e Doença. Universidade do Minho.

López, M. (2008). Cuidadores y cuidadoras: el efecto del género en el cuidado no profissional de los mayores. IMSERSO: Boletim sobre Envejecimiento, 35

Losada, A., Márquez-González, M., Peñacoba, C., GallagherThompson, D, \& Knight, B. (2007). Reflexiones en torno la atencíon a los cuidadores informales de personas con demencia y propuesta de una intervención interdisciplinar. Psicología Conductual, 15(1), 57-76.

Losada-Baltar, A., Trocóniz, M., Montorio-Cerrato, I., MárquezGonzález, M., \& Pérez-Rojo, G. (2004). Eficacia diferencial de las intervenciones psicoeducativas para cuidadores de familiares com demencia. Rev Neurol, 38(8), 701-708.

Losada-Baltar, A., \& Montorio-Cerrato, I. (2005). Pasado, presente y futuro de las intervenciones psicoeducativas para cuidadores familiares de personas mayores dependientes. Revista Española de Geriatría y Gerontología, 40(Supplement 2), 30-39.

Loss, Change \& Grief (2003). Caregiver stress: Journey of hearts. Retrieved February 24, 2003, from http://www. Caregiverstress.htm.

Lovell, B., \& Wetherell, M. A. (2011). The cost of caregiving: Endocrine and immune implications in elderly and non elderly caregivers. Neuroscience \& Biobehavioral Reviews.

Yee, JL., \& Schulz,R.(2000). Gender differences in psychiatric morbidity among family caregivers: a review and analysis, Gerontologist 2 (2000), pp. 147-164.

Mackey, R. A., Diemer, M. A., \& O'Brien, B. A. (2000). Conflict-management styles of spouses in lasting marriages. Psychotherapy, 37(2), 134-148

Marks, N.; Lambert, J. D. y Choi, H. (2002). Transitions to caregiving, gender, and psychological well-being: A prospective U.S. national study. Journal of Marriage and Family, 64, 657-667

Mittelman, M. S., Roth, D. L., Haley, W. E., \& Zarit, S. H. (2004). Effects of a caregiver intervention on negative caregiver appraisals of behavior problems in patients with Alzheimer's disease: results of a randomized trial. Journals of Gerontology Series B: Psychological Sciences and Social Sciences, 59(1), 27-34.

Miyamoto, Y., Tachimori, H., \& Ito, H. (2010). Formal Caregiver Burden in Dementia: Impact of Behavioral and Psychological Symptoms of Dementia and Activities of Daily Living. Geriatric Nursing, 31(4), 246-253.

Monin, J. K., \& Schulz, R. (2009). Interpersonal Effects of Suffering in Older Adult Caregiving Relationships. Psychology and Aging, 24(3), 681-695.

Moos, R. (1984). Coping with health crises In Handbook of clinical health psychological New York: Ed. Theodore Millon.

Morton, L. (2004). The Psychological Journey of an Alzheimer's Caregiver. LIAF - Newsletter of the Long Island Alzheimer's Foundation. 
Mulligan, E. A. (2010). Measuring predeath grief among dementia caregivers. Alzheimer's and Dementia, 6(4, Supplement 1), S314-S314.

Musil, C. M., Morris, D. L., Warner, C. B., \& Saeid, H. (2003). Issues in Caregivers' Stress and Providers' Support Research on Aging, 25(5), 505-526.

Nolan, M., Grant, G., \& Keady, J. (1998). Assessing the needs of family carers: a guide for practicioners. Brighton: Pavilion Publications.

Olshevski, J. L., Katz, A. D., \& Kight, B. G. (1999). Stress reduction for caregivers. Brunner/Mazel.

Paúl, C., \& Fonseca, A. M. (2005). Envelhecer em Portugal, psicologia saúde e prestação de cuidados ( $1^{\text {a }}$ ed.). Lisboa.

Pinquart, M., Sörensen, S., (2003). Differences between careguivers and noncareguivers in Psychological health and physical health: a meta - analysis. Psychology and Aging, 18 (2), 250 - 267

Ramos, M. (2001). Desafiar o desafio. Lisboa.

Ramos, M. (2005). Crescer em stress. Porto.

Ribeiro, J. (1998). Psicologia e Saúde. Lisboa: Instituto Superior de Psicologia Aplicada.

Rocca, W. A., Petersen, R. C., Knopman, D. S., Hebert, L. E., Evans, D. A., Hall, K. S., et al. (2011). Trends in the incidence and prevalence of Alzheimer's disease, dementia, and cognitive impairment in the United States. Alzheimer's and Dementia, 7(1), 80-93.

Sala, J. (2002). Família Y Demencia. Interventiones de apoyo y regulación. In, R., Alberca, \& S. López-Pousa (Coord.). Enfermedad de ALzheimer y otras demências, $2^{\mathrm{a}}$ Ed (pp.5070). Madrid: Ed. Médica Panamericana.

Serrano, P. (1996). Redacção e apresentação de trabalhos científicos. Lisboa: Relógio D’Água.

Schulz, R., Belle, S. H., Czaja, S. J., McGinnis, K. A., Stevens, A. y Zhang, S. (2004). Long-term care placement of dementia patients and caregiver health and well-being. JAMA, 292, 961-967.

Sotto Mayor, M. (2009). Quando a Pessoa a Cuidar tem Demência - A experiência Informal de Cuidar. Universidade do Porto, Porto.

Tschanz, J. T., Corcoran, C., Norton, M. C., Piercy, K., Rabins, P. V., Fauth, E., et al. (2010). Caregiver coping strategies predict cognitive decline in dementia: The Cache County Dementia Progression Study. Alzheimer's and Dementia, 6(4, Supplement 1), S346-S346.

Zarit, S. H., Charles, D., \& Irene, L. (2008). Diagnosis and management of caregiver burden in dementia. In handbook of Clinical Neurology (Vol. Volume 89, pp. 101-106): Elsevier.

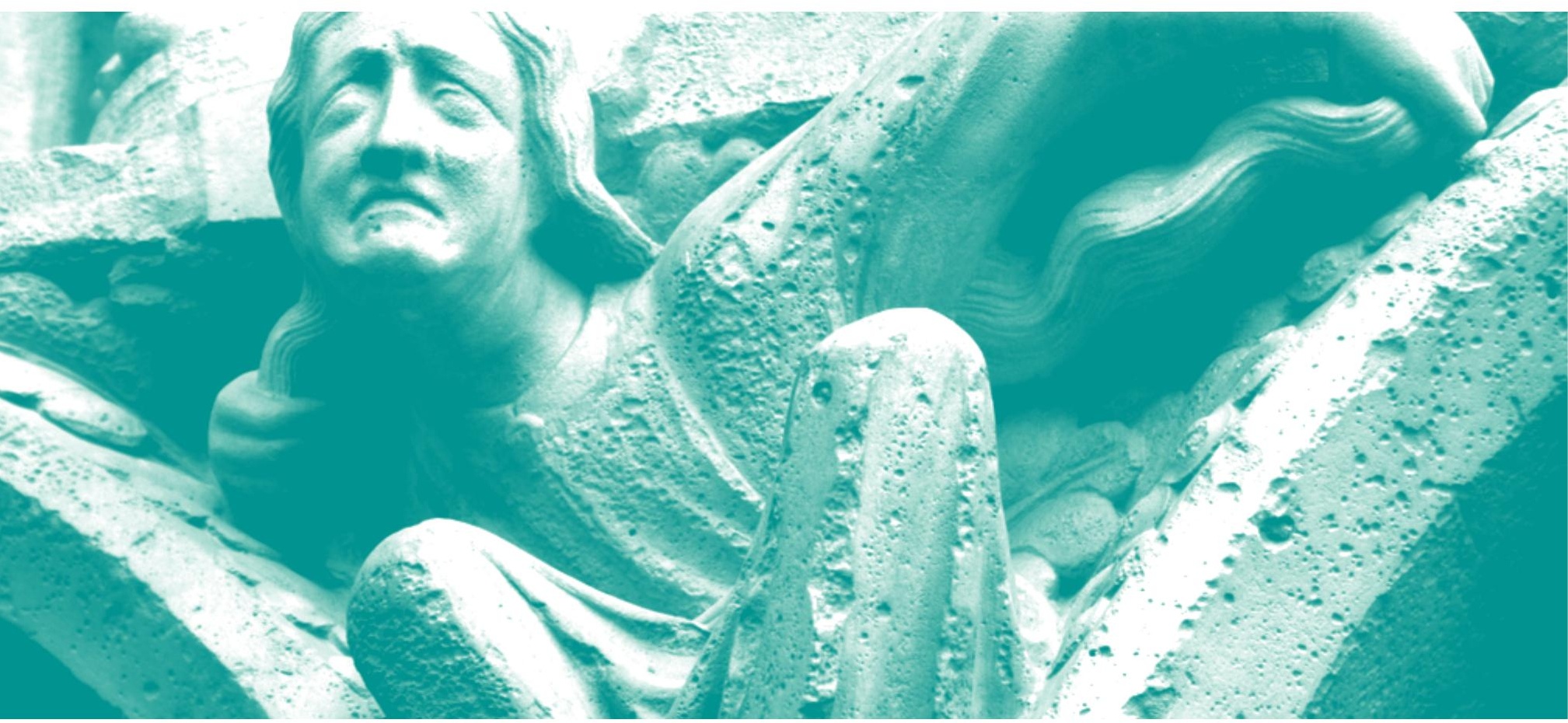

